\title{
Transplacental Route of Administration
}

National Cancer Institute

\section{Source}

National Cancer Institute. Transplacental Route of Administration. NCI Thesaurus. Code C38307.

Administration of a drug to an embryo in utero via systemic administration of the substance to the pregnant mother. Drugs cross the placenta through passive diffusion, active transport, pinocytosis and filtration. The properties of drugs that determine placental transfer by passive diffusion include molecular weight, pKa, lipid solubility and protein binding. Drugs with molecular weight of over $500 \mathrm{D}$ are transferred incompletely. Drug metabolism and uptake can be altered by placental enzymes. 